\title{
Characterizing and quantifying the effects of breast cancer therapy using mathematical modeling
}

\author{
Walter M. Gregory ${ }^{1}$ - Christopher J. Twelves ${ }^{2} \cdot$ Richard Bell $^{4} \cdot$ Stephen W. Smye $^{3}$. \\ Dena R. Howard ${ }^{1} \cdot$ Robert E. Coleman $^{5}$ - David A. Cameron ${ }^{6}$
}

Received: 22 September 2015/ Accepted: 7 January 2016/Published online: 19 January 2016

(c) The Author(s) 2016. This article is published with open access at Springerlink.com

\begin{abstract}
We designed a mathematical model to describe and quantify the mechanisms and dynamics of tumor growth, cell-kill and resistance as they affect durations of benefit after cancer treatment. Our aim was to explore how treatment efficacy may be related to primary tumor characteristics, with the potential to guide future trial design and appropriate selection of therapy. Assuming a log-normal distribution of both resistant disease and tumor doubling times generates disease-free survival (DFS) or invasive DFS curves with specific shapes. Using a multivariate mathematical model, both treatment and tumor characteristics are related to quantified resistant disease and tumor regrowth rates by allowing different mean values for the influence of different treatments or clinical subtypes on these two log-normal distributions. Application of the model to the CALGB 9741 adjuvant breast cancer trial showed that dose-dense therapy was estimated to achieve an extra 3/4 log of cell-kill compared to standard therapy,
\end{abstract}

Electronic supplementary material The online version of this article (doi:10.1007/s10549-016-3684-4) contains supplementary material, which is available to authorized users.

Walter M. Gregory

w.m.gregory@leeds.ac.uk

1 Leeds Institute for Clinical Trials Research (LICTR), University of Leeds, Clinical Trials Research House, 71-75 Clarendon Road, Leeds LS2 9JT, UK

2 Leeds Institute of Cancer and Pathology, University of Leeds, Leeds, UK

3 St. James's Academic Division of Medical Physics, University of Leeds, Leeds, UK

4 Deakin University, Geelong, Australia

5 Sheffield Cancer Research Centre, Sheffield, UK

6 Edinburgh University, Edinburgh, Scotland but only in patients with more rapidly growing ER-negative tumors. Application of the model to the AZURE trial of adjuvant bisphosphonate treatment suggested that the 5-year duration of zoledronic acid was adequate for ERnegative tumors, but may not be so for ER-positive cases, with increased recurrences after ceasing the intervention. Mathematical models can identify different effects of treatment by subgroup and may aid in treatment design, trial analysis, and appropriate selection of therapy. They may provide a more appropriate and insightful tool than the conventional Cox model for the statistical analysis of response durations.

Keywords Mathematical model - Adjuvant therapy · Bisphosphonates · Resistance $\cdot$ Cell-kill $\cdot$ Growth rate

\section{Introduction}

Mathematical models that incorporate information regarding tumor biology have the potential to provide mechanistic insights derived from trial data that cannot be gained by conventional statistical methods. We describe an approach that directly estimates the underlying biological parameters which generate particular DFS/IDFS curves.

\section{Previous work}

A previously published mathematical model $[1,2]$ related outcome durations to the amount of sub-clinical resistant disease and to tumor regrowth rates. Briefly, the model relates certain patterns in the shapes of DFS curves [1] to underlying quantity of undetectable resistant disease posttreatment, and the rate of tumor regrowth. Plateaus on the curves, gradients of the slopes, and the relationship 
between the height of the plateau and the delay on the curve before relapses start to occur, are all incorporated and explained by the model. It was hypothesized that DFS curves for faster growing tumors would have steeper slopes, and that the frequency and intensity of treatment should be matched to the aggressive growth of the tumor.

In the management of a number of early-stage solid cancers, primary surgical treatment removes the bulk primary tumor; the volume of any remaining disease being below the level of clinical detection. Based on tumor characteristics and pathological stage, adjuvant therapies may then be applied with the goal of reducing or eradicating this clinically undetectable residual disease. Clinical relapse occurs when tumor regrowth exceeds this level of detection. The model assumes that the component of this disease that is resistant to the adjuvant treatment employed is that which is destined to regrow and cause subsequent relapse, and that the volume of this resistant disease is log-normally distributed over the population of patients under consideration [1]. Adjuvant treatment is assumed to eradicate sensitive disease, but to be ineffective against resistant disease. In the event of the resistant disease being less than a given log volume (not necessarily 1 cell), the patient is assumed to be cured. Otherwise, the resistant disease is assumed to grow exponentially during and after treatment until relapse occurs, with the rate of regrowth being taken from a log-normal distribution of doubling times (Fig. 1). Similar model assumptions have been applied to other cancers $[1,5,6]$.

The model assumptions give rise to DFS curves with the desired shapes, enabling differences in curves to be ascribed to effects on either resistant disease burden at the end of treatment or subsequent regrowth rates. The "plateau" in the curve results from allowing for the possible extinction of the tumor when reduced below a given level. Rapidly growing tumors have a steeper curve slope. Curves with a pronounced delay before relapses start to occur are likely to have lower volumes of resistant disease, and therefore a higher plateau.

\section{New model developments}

The model has now been solved explicitly (see supplementary methods) and extended to a multivariate form. With the new model, prognostic factors can be related to components of both the regrowth rate and the level of undetectable resistant disease, potentially providing hypotheses for future tailoring of treatment. Measurable factors likely to be related to the volume of post-treatment resistant disease would include, as an example, primary tumor size at presentation [2], while those expected to correlate with tumor growth rates would include the histological grade of tumor, and markers of proliferation such as $\mathrm{Ki67}[3,4]$.
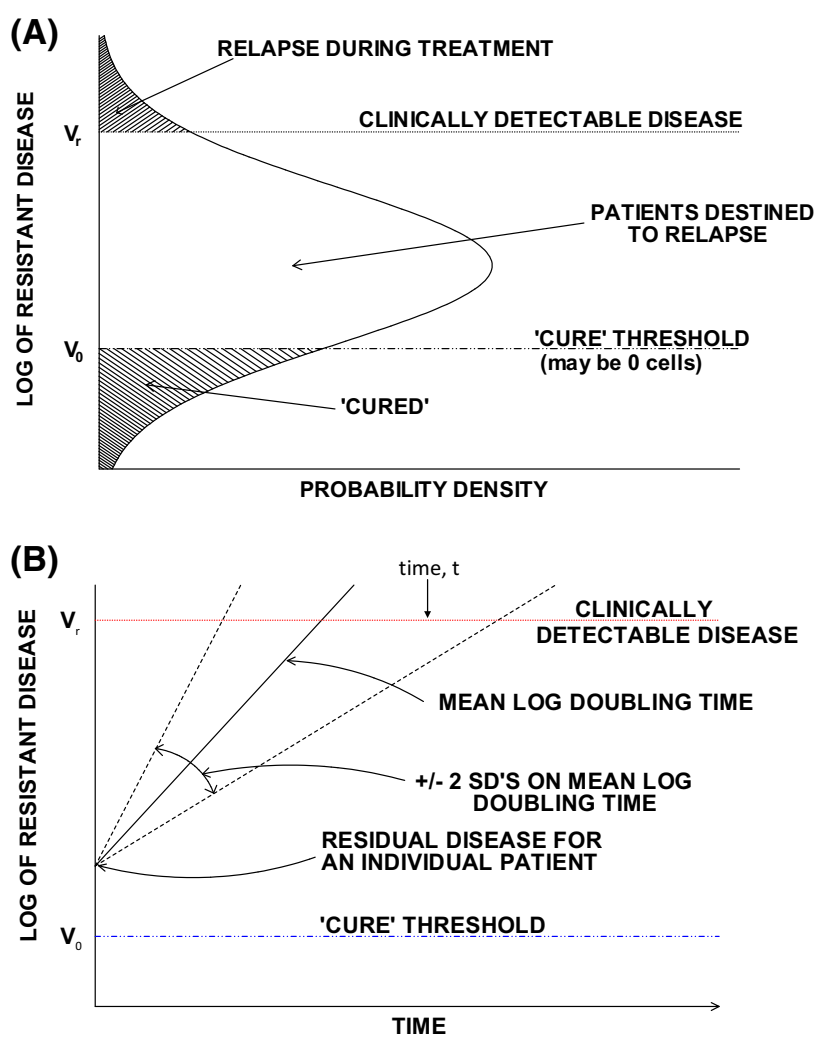

Fig. 1 Diagrammatic representation of the model: a assumed distribution of resistant disease after adjuvant treatment, $\mathbf{b}$ assumed pattern of regrowth rates of resistant disease

To demonstrate how the shape of the DFS curves relates to the underlying parameters, Fig. 2a, b show hypothetical curves from the original application [1] to treatments for acute myeloid leukemia (AML) [7] that produce purely resistant disease burden (effectively cell-kill) effects (Fig. 2a), compared with those that produce purely growth rate-related effects (Fig. 2b). In the latter case the curves diverge initially, but then gradually come back together, while in the former case divergence continues until relapses cease. The maximum likelihood algorithm effectively locates the closest such fits with a particular set of model assumptions (see supplementary methods).

This new multivariate mathematical model does not require proportional hazards, and therefore may also be more appropriate than the Cox model [8], for the analysis of these types of data.

\section{Applications}

Model applications to the CALGB 9741 and AZURE adjuvant breast cancer trials [9-11] are presented. The CALGB study employed a dose-dense chemotherapy schedule, based on the work of Norton and Simon [12, 13]. The dose-dense treatment, with shorter treatment intervals 
(A)

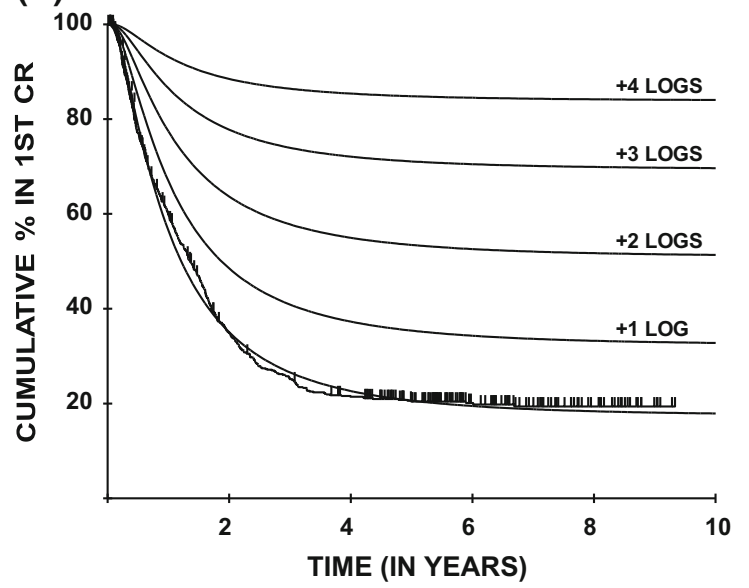

Fig. 2 Hypothesized effect on the duration of 1 st complete remission (CR) for patients in the MRC AML 8 trial of a 1-log improvements in eliminating (mean) resistant disease burden and $\mathbf{b}$ systematic slowing

would theoretically kill rapidly dividing cells more effectively, making it a good study in which to test the ability of the model to show differential effects on groups of patients with different tumor growth rates. In contrast, zoledronic acid, the bisphosphonate evaluated in the AZURE trial, differed in that its potential mechanism(s) of action was not understood and that bisphosphonate efficacy in the adjuvant setting was unproven. There was no a priori expectation that there would be any specific subgroup that would demonstrate a treatment effect. Information on several growth-related prognostic factors was, however, available affording the potential to evaluate treatment/growth rate interactions.

\section{Methods}

\section{The model}

The model, outlined in the introduction, is described mathematically in the supplementary methods section, with diagrammatic representation in Fig. 1. The new multivariate version assumes that the means of the two lognormal distributions can be related to both treatment and tumor characteristics in a linear multiplicative fashion, thereby producing different DFS curves for different treatment/prognostic groups.

\section{Application of the model}

The model is applied to DFS times, complete and censored, and a set of treatment/prognostic factors are then examined for their relationship to these durations of response, via

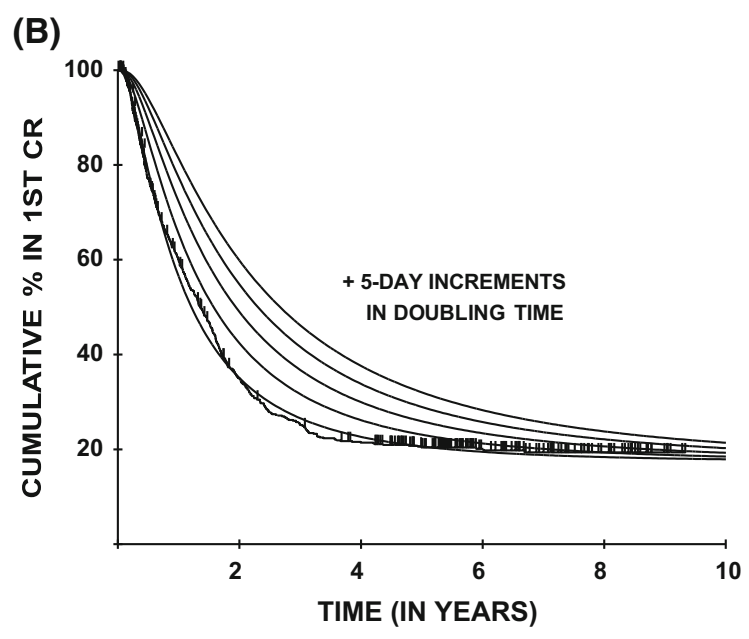

down of the doubling time (in 5 day increments from 16.6 to 36.6 days). The actuarial reference curve is also shown

effects on resistant disease and growth rates, using a forward stepwise approach (see supplementary methods). Factors can be included for their effects both on growth rates and/or resistant disease. The significance of a covariate, if required, can be evaluated by testing the pair of parameters together, namely resistant disease and doubling time, in the new model. Related computer programs are available (see supplementary methods).

\section{Model assumptions}

The model assumes exponential growth. Alternative growth rate assumptions, such as the gompertzian model proposed by Norton [14], were considered; the model is, however, attempting to estimate the growth rate of subclinical disease, where the gompertzian model is less relevant. The assumption of a log-normal distribution of growth rates is consistent with other findings $[15,16]$.

The volume of resistant disease after treatment is assumed to be independent of its growth rate, consistent with results from an independent set of data from CLL (data not shown). The original rationale for assuming a lognormal distribution of resistant disease $[1,5,6]$ was related to a Goldie/Coldman mathematical model where random spontaneous mutations conferred drug resistance over a tumor's lifetime [2]; this assumption was shown to lead to a log-normal distribution of resistant tumor at presentation [5]. The situation is somewhat different with operable breast cancer treated initially by surgery, with or without radiotherapy, where the local disease has been effectively eliminated but the cancer may have disseminated through vascular or lymphatic channels [16]. Before diagnosis, as the tumor increases in size, the likelihood of occult distant 
metastases developing increases. Thus, we can assume a random spontaneous chance of mutations to a metastatic phenotype developing and cells becoming disseminated, which increases over time, with a similar pattern of mutation to resistance; this results in a log-normal distribution of, in this case, disseminated resistant disease at presentation.

Note that it is assumed that $\geq 10^{9}$ cells are clinically detectable, and that all these cells need to be eliminated to eradicate the disease. The choice of this level, which is the same for all covariates, does not affect the shape of the distribution of growth rates or the fitting process; it merely affects the doubling time estimates. The model estimates for doubling times under this assumption are consistent with those reported in breast cancer [15].

\section{Model-based DFS curves and comparison with the Cox model}

Model fits for both models can be shown by deriving an estimated survival curve for each prognostic category, taking into account the mix of treatment and prognostic factors that determine a specific resistant disease volume and growth rate, averaging these survival figures over a group of patients, for example those with ER-positive disease, then validating this by comparison with the actuarial curve (see Figs. 3, 4, and 5 and supplementary Fig. 1). Akaike's Information Criterion (AIC) [17] allows for the (four) extra parameters in this new model in comparison with the partial likelihood derived from the Cox model and facilitates an order-of-magnitude comparison of the fits of the two models (see supplementary methods).

\section{Application data/studies}

CALGB study 9741 treated 1973 women with node-positive breast cancer. One of the two randomizations in this study tested dose-dense chemotherapy comprising doxorubicin, cyclophosphamide, and paclitaxel given every two weeks versus a conventional schedule given every three weeks $[9,10]$. Berry et al. [10] broke down the results by ER status demonstrating a $24 \%$ [95 \% CI 1-42\%] reduction in risk of recurrence for women with ER-negative tumors, but a non-significant risk reduction of $8 \%$ [95 \% CI 20-29 \%] in ER-positive cases at a median follow-up of 6 years.

The AZURE trial [11] tested the hypothesis that bisphosphonate treatment may reduce rates of recurrence and death in 3360 women with high risk early breast cancer, with axillary lymph node metastasis (N1) or T3/T4 primary tumors. Consenting patients were randomized to receive standard adjuvant systemic therapy with or without zoledronic acid following local treatment. Zoledronic acid treatment was planned for 5 years duration. The median follow-up at the time of reporting was 7 years. Zoledronic acid was effective only in postmenopausal women $(n=1041)$, with an IDFS hazard ratio of.75 [95 \% CI 59.96]. Efficacy in this subgroup was later verified in a metaanalysis of other studies [18]. This analysis focuses on invasive disease-free survival (IDFS).

\section{Reconstructing DFS data}

Published DFS graphs from CALGB 9741 [10] were scrutinized and individual survival times were successfully derived, ensuring that all $p$-values, confidence intervals (CIs), numbers of events, median DFS times, and durations of patient follow-up matched those reported. For the AZURE trial individual patient data were provided by the Leeds Clinical Trials Research Unit.

\section{Results}

\section{CALGB 9741}

The associated DFS curves for study 9741, along with the model fits, are given in Fig. 3a. From the model results dose-dense therapy was estimated to produce 3/4 log of additional cell-kill in women with ER-negative disease, which equates to an additional $10 \%$ of these women being cured. The associated distribution of resistant disease is shown in Fig. 3b, and comparison with the Cox model DFS estimates are shown in Fig. 3c. The model clearly attributed the difference in ER status DFS curves to a large difference in mean doubling times: 31 days for ER-negative women compared with 84 days for ER-positive women (Fig. 3d).

\section{Azure}

Model analyses again attributed ER status differences in IDFS to a difference in growth rates, with estimated mean doubling times of 39 and 114 days for ER-negative and ER-positive women, respectively (shown graphically in supplementary Fig. 1). In support of this finding ER status was also highly correlated with tumor grade (see Table 1). Model fits to the two treatment arms in postmenopausal women are shown in Fig. 4a. The curves appear to show a possible fall-off after 5 years, which coincides with stopping treatment, and so the model was re-run using data up to 5 years only, with data after this time being censored. The model fits are shown in Fig. 4b. Since this is a mathematical model, it is possible to extrapolate these curves beyond the 5 years of data that were used to fit the curves, as in Fig. 4c to predict IDFS with continuation of 
(A)

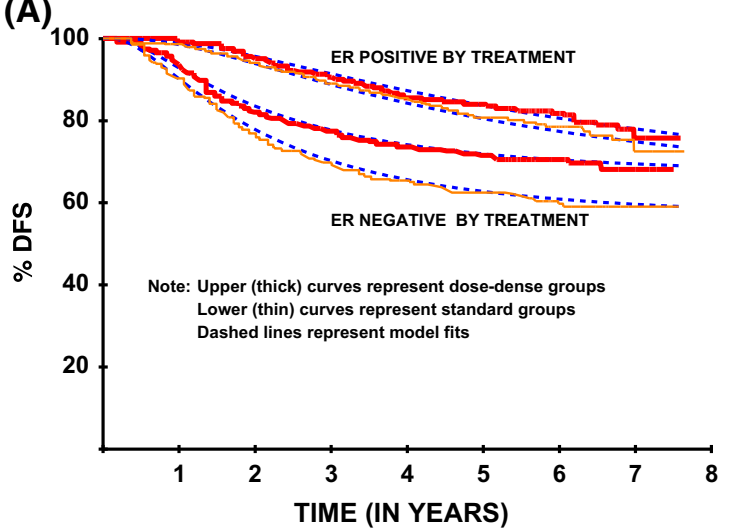

(C)

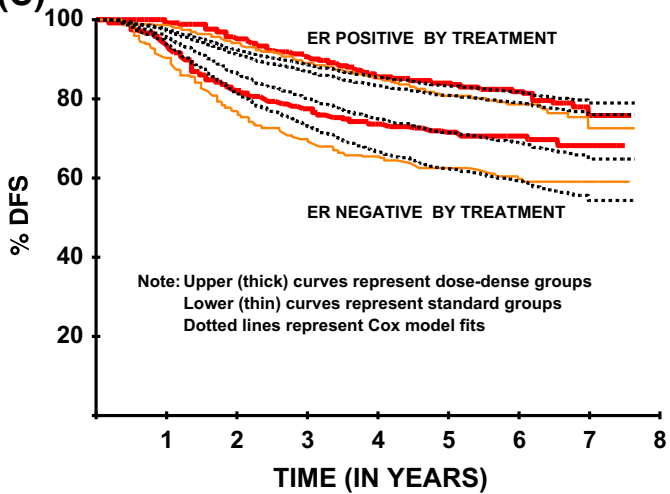

Fig. 3 Mathematical model and Cox model results for CALGB trial 9741, looking at the effects of dose-dense treatment by ER status: a mathematical model fits to DFS curves, b mathematical model

Table 1 Correlation between histological grade and ER status in the AZURE trial

\begin{tabular}{lcccl}
\hline & ER + ve & ER - ve & $\%$ ER + ve & $\chi^{2}$ (trend) \\
\hline Grade 1 & 86 & 0 & 100 & 157.2 \\
Grade 2 & 410 & 44 & 90 & $(p<.0001)$ \\
Grade 3 & 265 & 202 & 57 & \\
\hline
\end{tabular}

treatment. For the zoledronic acid group there was a marked divergence from the predictions starting immediately at the cessation of treatment at 5 years (Fig. 4d). However, the divergence occurs only in women with ERpositive disease (Fig. 5a), not those with ER-negative disease (Fig. 5b). For the control arm the predictions were much more accurate, only beginning to diverge, if at all, at about 7 years, when the numbers at risk are small and the confidence intervals in the IDFS curves are wide. To test whether these differences from model predictions after 5 years are chance findings IDFS times were generated to match the modeled pattern beyond 5 years and compared with the actual data beyond 5 years using a landmark analysis beginning at 5 years. This revealed a highly significant difference between actual data and predicted data
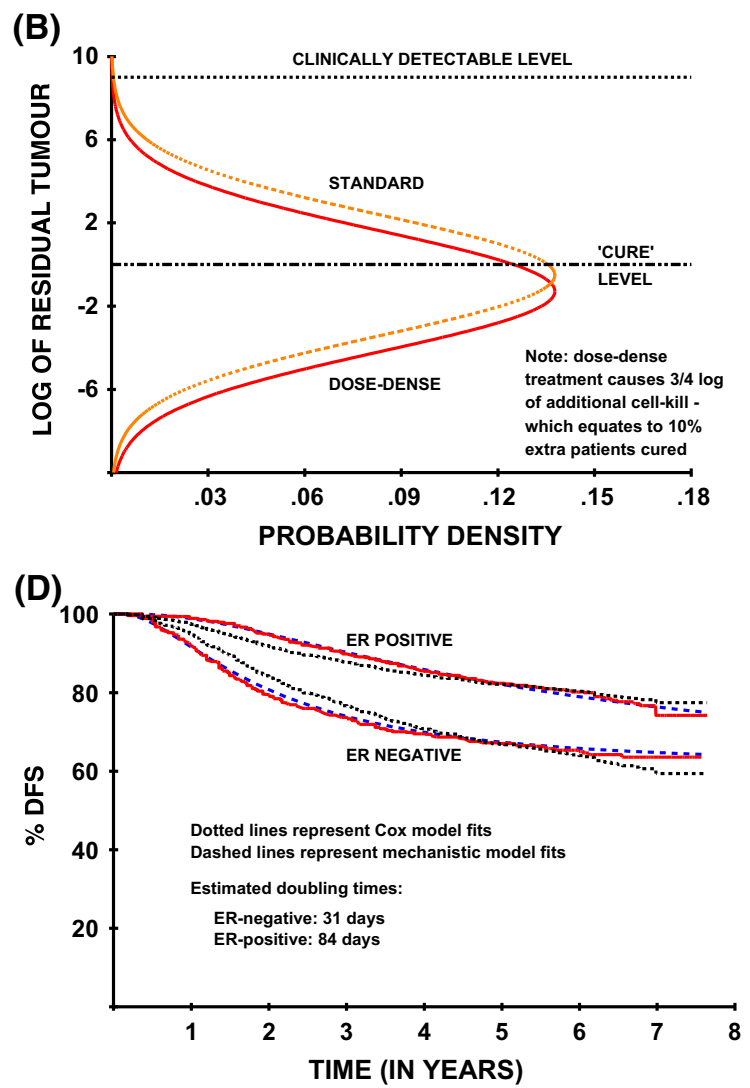

estimates of resistant disease after treatment for ER-negative patients by dose-dense treatment, c Cox model fits to DFS curves, and d mathematical model and Cox model fits to ER status DFS curves

in the zoledronic acid group ( $p=.0014$, Wilcoxon test) compared with a non-significant difference in the control group ( $p=.22$, Wilcoxon test) confirming that this is not simply a chance effect.

Stepwise biological model results for tumor grade, size, nodal status, and ER status are given in detail in supplementary Table 1 . Hazards were clearly not proportional for ER status or for histological grade $(p<.001)$ resulting in poor fits for the Cox model, whereas, in contrast, the new model incorporates such effects explicitly and exhibits good fits (see supplementary Fig. 1). AIC differences confirm these interpretations, and are presented in the supplementary methods. The fits for the two models for $\mathrm{T}$-stage and for the number of involved lymph nodes were similar.

\section{Discussion}

This new biologically based model demonstrates that it is possible to determine, in a logical and structured fashion, interrelationships between treatment and biological factors of the primary tumor, via their effects on the crucial 
(A)

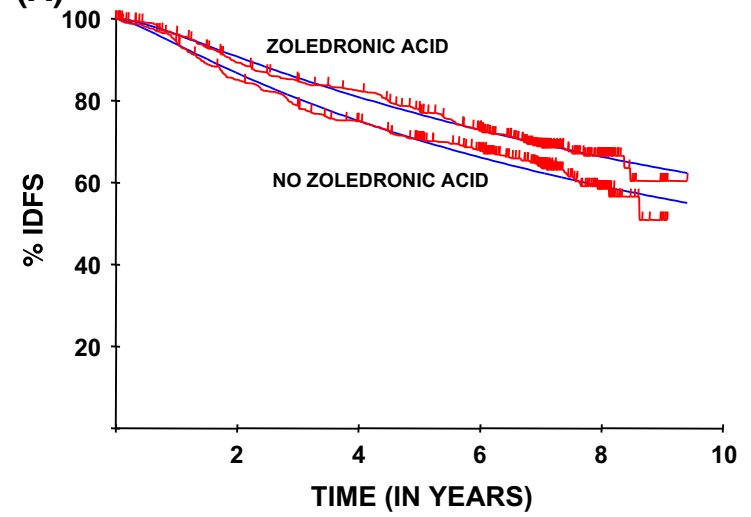

(C)

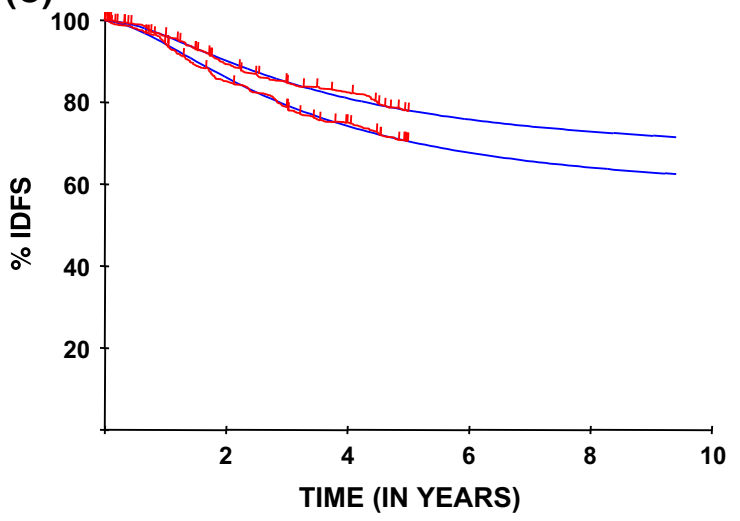

Fig. 4 Mathematical model results for AZURE trial looking at treatment with or without zoledronic acid, with model fits and projected model fits based on the first 5 years' worth of data only: a model fits to zoledronic acid/no zoledronic acid IDFS curves, b model fits to zoledronic acid/no zoledronic acid IDFS curves based on first 5 years' worth of data only, $\mathbf{c}$ model fits to zoledronic acid/no

parameters of resistant sub-clinical tumor burden and growth rates.

The model we developed resulted in additional results and hypotheses over and above those obtained from conventional trial analyses. Women with ER-negative breast cancer are estimated to have more rapid tumor growth rates; they may, therefore, receive benefit from more frequent chemotherapy, as was demonstrated with accelerated chemotherapy in the CALGB 9741 study [10]. Further evidence to support this comes from an analysis of the CALGB 8541 trial, which tested lower doses/shorter durations of adjuvant CAF chemotherapy [10]. In that study, for women with ER-negative disease, who would be expected to have more rapidly proliferating tumors, 4 cycles of standard CAF chemotherapy was superior to 6 cycles of lower dose CAF; by contrast, for those with more slowly proliferating ER-positive disease lower dose CAF given for a longer duration was equally effective [10]. Differential effects of treatment by ER status are
(B)

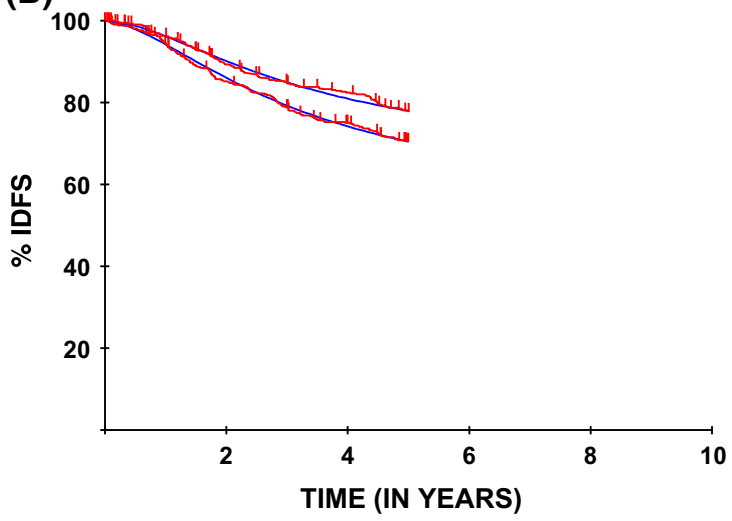

(D)

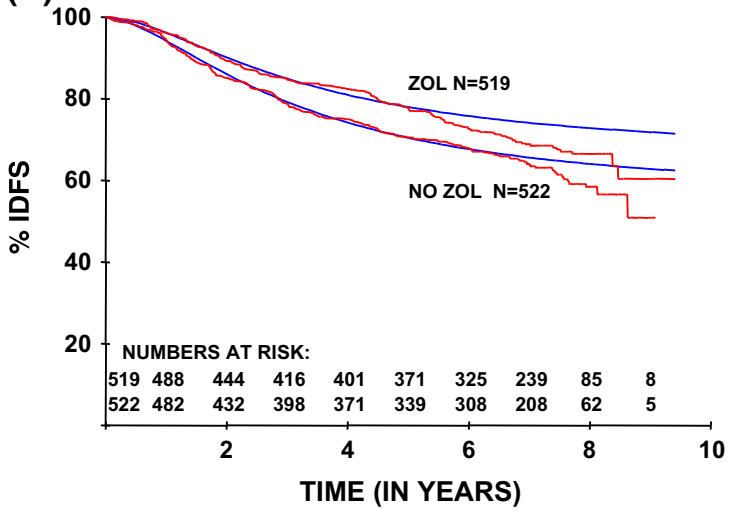

zoledronic acid IDFS curves based on first 5 years' worth of data only but projected out to current follow-up, $\mathbf{d}$ model fits to zoledronic acid/ no zoledronic acid IDFS curves based on first 5 years' worth of data only projected out to current follow-up compared with actual IDFS outcomes

particularly pronounced in both breast cancer datasets, though similar personalizing of therapy could be considered within different histological grade, or genomic subgroups. This model will outperform standard models such as the Cox model where factors relate to growth rate differences, which are unlikely to fit the proportional hazards assumption.

What distinguishes this new model from the classical statistical approach of the Cox model is the incorporation of an a priori understanding of biological mechanisms; it does not require the often flawed assumption of proportional hazards, yet produces substantially better model fits in these breast cancer DFS/IDFS datasets than does the equivalent Cox model (see Fig. 3 and supplementary Fig. 1). Interactions with time, and stratification by nonproportional hazards variables such as ER status, can, of course, be incorporated into Cox models to avoid the assumption that hazard ratios are constant across time, but at the cost of added complexity often making the results 


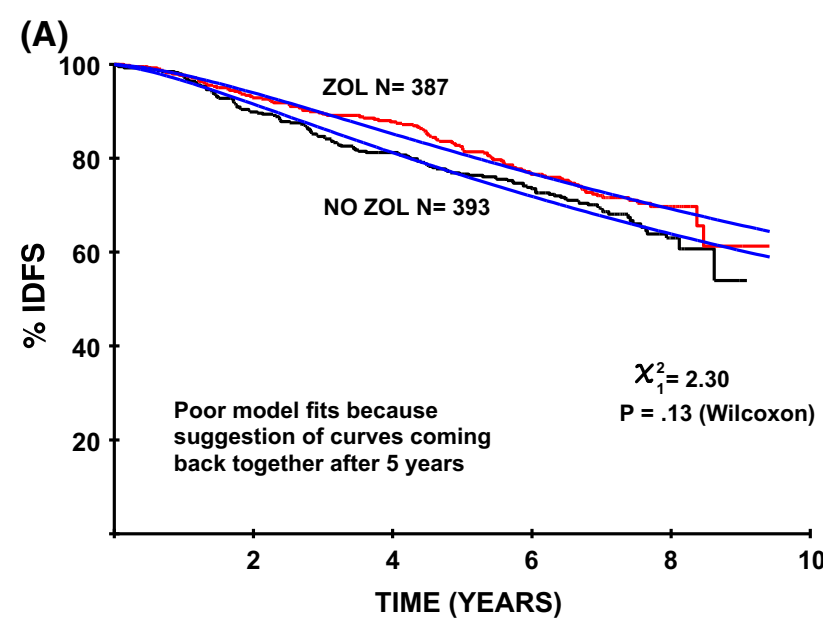

(B)

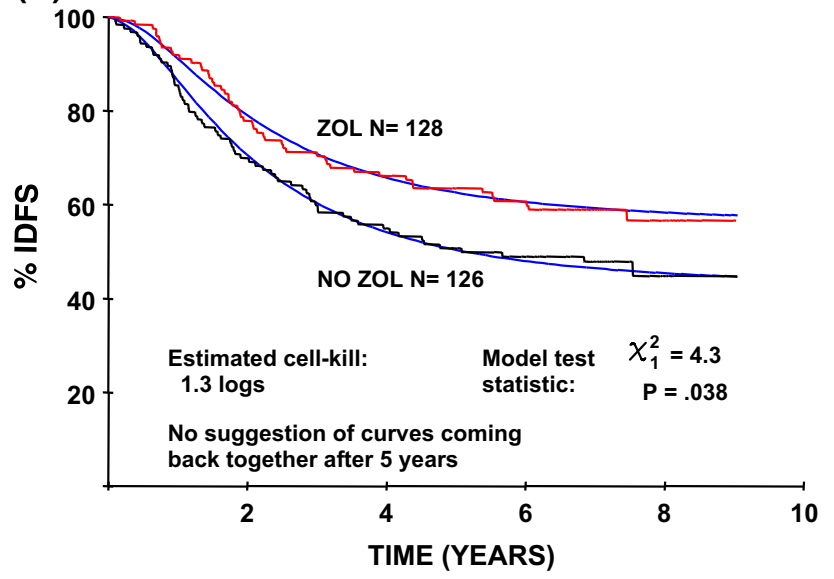

Fig. 5 Mathematical model results for AZURE trial looking at treatment with or without zoledronic acid, broken down by ER status: a zoledronic acid/no zoledronic acid IDFS curves for ER-positive women only, and b zoledronic acid/no zoledronic acid IDFS curves for ER-negative women only, both with associated model fits which are based on the whole dataset, not just the first 5 years

difficult to understand. This model, in contrast, produces results that relate to the very biological parameters that the researchers are usually trying to affect in the trial design, and are therefore meaningful and directly interpretable. The distinction between these two approaches is significant since our model offers the prospect of developing a clinically useful framework for cancer treatment that incorporates the increasingly sophisticated understanding of tumor biology gained from experimental studies. Such a framework could include features such as measures of tumor genome heterogeneity [19] and consequent evolutionary dynamics [20]. The former might be reflected, for example, in the parameters describing resistant volume and growth rate. Such models could incorporate factors such as pharmacokinetic parameters and have the potential to inform personalized treatment regimens $[21,22]$. Because this approach is mathematical, it offers a rigorous and quantitative approach to integrating various aspects of tumor biology and determining the consequences for therapy [23].

Applying the insights gained from the CALGB study, we can consider the AZURE results in the light of factors relating to tumor growth rate. Firstly, the correlation between ER status and tumor grade again supports the conclusion derived from the model analyses that ER-negative women have faster growing tumors. Then considering the treatment effect by ER status, a fall-off in the IDFS curves at 5 years is only seen in the women with ERpositive disease not in those with ER-negative disease (Fig. 5a, b). We have not found departures such as this to be common, and we have now had considerable experience of using this model across a range of cancers. This analysis therefore suggests that 5 years of zoledronic acid treatment is adequate in women with the faster growing ER-negative tumors, but perhaps insufficient for those with more slowly proliferating ER-positive tumors who continue to relapse at a significant rate well beyond the 5 year time point. This conclusion would not have been forthcoming from the Cox model, which does not deal well with growth rate effects. Although zoledronic acid has a long half-life in bone, there may be a threshold of residual activity and inhibition of bone turnover required for benefit to occur. Tumor dormancy is also a much more prominent feature in ER-positive disease and may well be of relevance to these findings, with $>50 \%$ of relapses beyond 5 years reflecting the late, unexplained, emergence of disseminated tumor cells from the dormant state [24]. Continuous long-term application of treatment appears necessary to prevent this re-awakening of quiescent ER-positive cells unlike the highly proliferative ER-negative disseminated cells which are typically either eradicated by adjuvant treatments or initiate clinically detectable metastases over a much shorter timeframe (typically $<5$ years). It is intriguing that recent data suggest longer duration of treatment may also benefit patients receiving adjuvant tamoxifen therapy [25-27]. We do not, therefore, assume this effect to be specific to bisphosphonates. The findings are consistent with the long duration of therapy that is now the standard of care in hormone-receptor positive breast cancer, with 5 years as a minimum standard, and with several studies supporting extended therapy to 10 years $[28,29]$. Thus, the model may lead to the generation of novel treatment strategies, as did the original univariate model [1] in acute lymphoblastic leukemia, Hodgkin's disease, multiple myeloma, and breast cancer [6].

It is well recognized that cancers are not homogenous with regard to biology and natural history. This was first documented in breast cancer with the recognition that women with ER-positive disease were more at risk of late relapse than those with ER-negative disease. As molecular 
and genetic understanding grows in this and other cancers, identification of patients with different outcomes will be increasingly possible. The model demonstrates that it is possible to determine these differences, and their potential interactions with treatment, by modeling the "shape" of the DFS/IDFS curves. Furthermore, with that insight, the mathematical model allows predictions to be made of the long-term benefit beyond the period of follow-up of the study and generate hypotheses that can be tested in prospective randomized trials. This model, as with all such models, represents a considerable simplification of tumor dynamics, since it does not, at present, explicitly incorporate current understanding of immune signaling, angiogenesis, stem-cell subpopulations, and other important host-tumor interactions, but unless they are thought to act other than on the net tumor doubling time and resistant disease volume, their effects could still be estimated. As more data are generated on the biological and genetic characteristics of cancers, this approach may be further developed and additional complexity included as required.

Concepts derived from mathematical modeling have been productive in generating new trials and treatment approaches, for instance of alternating non-cross resistant drug combinations as proposed by Goldie et al. [30], or hypotheses regarding proportional cell-kill and its exploitation as suggested by Skipper et al. [31, 32]. More recently, and since the publication of the univariate version of this model, Norton [14] and Day et al. [33] used the Gompertzian model of tumor growth in breast cancer and derived fits to observed response duration curves using simulation techniques [14], and numerical integration [33]. The Gompertzian model provided the background for the dose-dense chemotherapy schedules $[9,10]$ which have since been tested in clinical trials $[11,12]$ and implemented in clinical practice. As with all pre-clinical hypotheses in cancer, not all of these insights have been confirmed in prospective clinical studies, but the mathematical model described herein is a continuation of these lines of thought, designed to quantify and add scientific rigor to these concepts by analytically fitting such a model. This brings with it related statistical techniques such as significance testing and confidence intervals, as well as enabling a multivariate form to become a practical tool for trial data interpretation. This approach may be especially relevant in the era of rationally designed targeted therapies, defining subgroups where therapeutic interactions occur. The application of the model warrants confirmation in future prospectively designed clinical trials.

Funding Research support was received from Novartis in the form of an academic unrestricted educational Grant.

\section{Compliance with ethical standards}

Conflicts of interest Author Walter Gregory has received consultancy and honoraria for lectures for Celgene and Janssen. Author David Cameron has undertaken consultancy for Novartis (uncompensated). Author Robert Coleman has received research funding from Amgen, Novartis, Celgene, and Bayer, and has provided expert testimony for Novartis.

Open Access This article is distributed under the terms of the Creative Commons Attribution-NonCommercial 4.0 International License (http://creativecommons.org/licenses/by-nc/4.0/), which permits any noncommercial use, distribution, and reproduction in any medium, provided you give appropriate credit to the original author(s) and the source, provide a link to the Creative Commons license, and indicate if changes were made.

\section{References}

1. Gregory WM, Richards MA, Slevin ML, Souhami RL (1991) A mathematical model relating response durations to amount of sub-clinical resistant disease. Cancer Res 51:1210-1216

2. Goldie JH, Coldman AJ (1979) A mathematical model for relating the drug sensitivity of tumors to their spontaneous mutation rate. Cancer Treat Rep 63:1727-1731

3. Gerdes J, Dallenbach F, Lennert K, Lemke H, Stein H (1984) Growth fractions in malignant non-Hodgkin's lymphoma as determined in situ by the monoclonal antibody Ki67. Haematol Oncol 2:365-371

4. Barnard N, Hall P, Lemoine N, Kodar N (1987) Proliferative index in breast carcinoma determined in situ by Ki67 immunostaining and it's relationship to clinical and pathological variables. J Pathol 152:287-295

5. Gregory WM (1993) The evaluation of durations of response to cancer treatments. Dissertation, University College London

6. Gregory WM (1992) Cell proliferation and the principles of cancer therapy. In: Hall PA, Levison DA, Wright NA (eds) Assessment of cell proliferation in clinical practice. SpringerVerlag, London, pp 193-205

7. Rees JKH, Gray RG, Swirsky D, Hayhoe FGJ (1986) Principal results of the medical research council's 8th acute myeloid leukaemia trial. Lancet 2:1236-1241

8. Cox DR (1972) Regression models and life tables. J Royal Stat Soc Ser B 34:187-220

9. Citron ML, Berry DA, Cirrincione C, Hudis C, Winer EP, Gradishar WJ et al (2003) Randomized trial of dose-dense versus conventionally scheduled and sequential versus concurrent combination chemotherapy as postoperative adjuvant treatment of node positive primary breast cancer: first report of intergroup trial C9741/cancer and leukaemia group B trial 9741. J Clin Oncol 21:1431-1439

10. Berry DA, Cirrincione C, Henderson IC, Citron ML, Budman DR, Goldstein LJ et al (2006) Estrogen-receptor status and outcomes of modern chemotherapy for patients with node-positive breast cancer. JAMA 295:1658-1667

11. Coleman RE, Marshall H, Cameron D, Dodwell D, Burkinshaw R, Maccon Keane M et al (2011) Breast-cancer adjuvant therapy with zoledronic acid. N Engl J Med 365:1396-1405

12. Norton L, Simon R (1977) Tumor size, sensitivity to therapy, and design of treatment schedules. Cancer Treat Rep 61:1307-1317

13. Norton L, Simon R (1986) The Norton-Simon hypothesis revisited. Cancer Treat Rep 70:163-169 
14. Norton L (1988) A gompertzian model of human breast cancer growth. Cancer Res 48:7067-7071

15. Shackney SE, McCormack GW, Cuchural Jnr GJ (1978) Growth rate patterns of solid tumors and their relation to responsiveness to therapy: an analytical review. Ann Intern Med 89:107-121

16. Tubiana M, Pejovic MH, Koscielny S, Chavaudra N, Malaise E (1989) Growth rate, kinetics of tumor cell proliferation and longterm outcome in human breast cancer. Int J Cancer 44:17-22

17. Akaike H (1974) A new look at the statistical model identification. IEEE Trans Autom Control 19:716-723

18. Gregory W, Marshall H, Bell R, Cameron DA, Coleman RE (2012) Adjuvant zoledronic acid (ZOL) in postmenopausal women with breast cancer and those rendered postmenopausal: results of a meta-analysis. J Clin Oncol. 30: (suppl; abstr 513)

19. Zhang Q, Austin RH (2012) Physics of cancer: the impact of heterogeneity. Ann Rev Condensed Matter Phy 3:363-382

20. Bozic I, Reiter JG, Allen B, Antal T, Chatterjee K, Shah P, et al (2013) Evolutionary dynamics of cancer in response to targeted combination therapy. eLife; 2:e00747

21. Gregory WM, Reznek RH, Hallett M, Slevin ML (1990) Using mathematical models to estimate drug resistance and treatment efficacy via CT scan measurements of tumor volume. Br J Cancer 62:671-675

22. Birkhead BG, Gregory WM, Slevin ML, Harvey VJ (1986) Evaluating and designing cancer chemotherapy treatments using mathematical models. Eur J Cancer 22:3-8

23. Hofree M, Shen JP, Carter H, Gross A, Ideker T (2013) Networkbased stratification of tumor mutations. Nat Methods 10(11):1108-1115

24. Ren G, Esposito M, Kang Y (2015) Bone metastasis and the metastatic niche. J Mol Med 93:1203-1212

25. Higgins MJ, Liedke PE, Goss PE (2013) Extended adjuvant endocrine therapy in hormone dependent breast cancer: the paradigm of the NCIC-CTG MA.17/BIG 1-97 trial. Crit Rev Oncol Hematol 86(1):23-32

26. Davies C, Pan H, Godwin J, Gray R, Arriagada R, Raina V et al (2013) Long-term effects of continuing adjuvant tamoxifen to 10 years versus stopping at 5 years after diagnosis of oestrogen receptor-positive breast cancer: ATLAS, a randomised trial. Lancet 381:805-816

27. Gray RG, Rea D, Handley K, Bowden SJ, Perry P, Earl HM, et al (2013) ATTOM: Long-term effects of continuing adjuvant tamoxifen to 10 years versus stopping at 5 years in 6953 women with early breast cancer. J Clin Oncol. 31: (suppl; abstr 5)

28. Strasser-Weippl K, Badovinac-Crnjevic T, Fan L, Goss PE (2013) Extended adjuvant endocrine therapy in hormone-receptor positive breast cancer. Breast 22(Suppl 2):S171-S175

29. Early Breast Cancer Trialists' Collaborative Group (EBCTCG), Davies C, Godwin J, Gray R, Clarke M, Cutter D et al (2011) Relevance of breast cancer hormone receptors and other factors to the efficacy of adjuvant tamoxifen: patient-level meta-analysis of randomised trials. Lancet 378:771-784

30. Goldie JH, Coldman AJ, Gudauskas GA (1982) Rationale for the use of alternating non-cross-resistant chemotherapy. Cancer Treat Rep 66:439-449

31. Skipper HE, Schabel FMJ, Wilcox WS (1964) Experimental evaluation of potential anticancer agents. XIII. On the criteria and kinetics associated with 'curability' of experimental leukemia. Cancer Chemother Rep 35:1-111

32. Skipper HE, Schabel FMJ, Wilcox WS (1967) Experimental evaluation of anticancer agents. XXI. Scheduling of arabinosylcytosine to take advantage of its S-phase specificity against leukemia cells. Cancer Chemother Rep 51:125-165

33. Day R, Shackney S, Peters W (2005) The analysis of relapse-free survival curves: implications for evaluating intensive systemic adjuvant treatment regimens for breast cancer. $\mathrm{Br} \mathrm{J}$ Cancer 92:47-54 